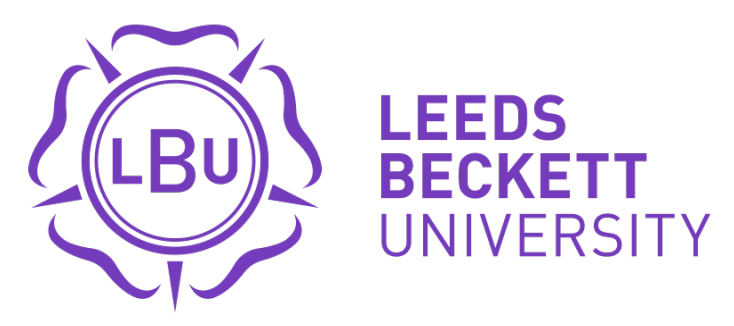

Citation:

Rongen, F and Till, K and Mckenna, J and Tee, JC and Cobley, S (2020) Psychosocial outcomes associated with soccer academy involvement : Longitudinal comparisons against aged matched school pupils. Journal of Sports Sciences. ISSN 1466-447X DOI: https://doi.org/10.1080/02640414.2020.1778354

Link to Leeds Beckett Repository record:

https://eprints.leedsbeckett.ac.uk/id/eprint/6787/

Document Version:

Article (Accepted Version)

This is an Accepted Manuscript of an article published by Taylor \& Francis in Journal of Sports Sciences on 16 June 2020, available online: http://www.tandfonline.com/10.1080/02640414.2020.1778354

The aim of the Leeds Beckett Repository is to provide open access to our research, as required by funder policies and permitted by publishers and copyright law.

The Leeds Beckett repository holds a wide range of publications, each of which has been checked for copyright and the relevant embargo period has been applied by the Research Services team.

We operate on a standard take-down policy. If you are the author or publisher of an output and you would like it removed from the repository, please contact us and we will investigate on a case-by-case basis.

Each thesis in the repository has been cleared where necessary by the author for third party copyright. If you would like a thesis to be removed from the repository or believe there is an issue with copyright, please contact us on openaccess@leedsbeckett.ac.uk and we will investigate on a case-by-case basis. 


\section{Psychosocial outcomes associated with soccer academy involvement: Longitudinal comparisons against aged matched school pupils}

Fieke Rongen ${ }^{\mathrm{a}}$, Jim McKenna ${ }^{\mathrm{a}}$, Stephen Cobley ${ }^{\mathrm{b}}$, Jason C. Tee ${ }^{\mathrm{a}, \mathrm{c}}$, and Kevin Till ${ }^{\mathrm{a}}$

${ }^{a}$ Institute for Sport, Physical Activity and Leisure, Leeds Beckett University, Leeds, West Yorkshire, United Kingdom, ${ }^{b}$ : Faculty of Health Science, University of Sydney, Sydney, Australia; ${ }^{c}$ : Department of Sport Studies, Faculty of Applied Sciences, Durban University of Technology, South Africa.

Address for correspondence:

Fieke Rongen,

Room 113, Cavendish Hall,

Institute for Sport, Physical Activity and Leisure,

Headingley Campus,

Leeds Beckett University,

West Yorkshire,

LS6 3QS

Telephone: +44-113-812 3570

Email: f.rongen@leedsbeckett.ac.uk 


\title{
Psychosocial outcomes associated with soccer academy involvement: Longitudinal comparisons against aged matched school pupils
}

\author{
Abstract: \\ Despite literature highlighting numerous risks to the healthy psychosocial \\ development of youth elite academy soccer players, little of this research is based \\ on high quality research designs. This study employed a prospective longitudinal \\ cohort design to track psychosocial outcomes of academy involvement within \\ male youth elite soccer players ( $n=33$, U12-U16 age groups) compared to age- \\ matched soccer-active school pupils $(n=44)$ over 12 months. Participants \\ completed questionnaires assessing the most commonly raised psychosocial \\ concerns at four equally spaced data collection periods (T1-T4). Repeated \\ measures multivariate analysis of variance (MANOVAs) indicated that, over the \\ year, both groups reported a healthy and improving stress and recovery balance, \\ as well as positive and stable needs satisfaction and physical, psychological and \\ social well-being. Academy players reported stable positive school-related \\ quality of life, whereas school pupils reported increases from T3 to T4. Academy \\ players reported consistent significantly higher total athletic identity and \\ exclusivity of identity. Findings suggest that many concerns around negative \\ psychosocial impacts of soccer academy involvement did not materialise in this \\ context. However, heightened athletic identities remained a concern.
}

Keywords: Elite youth soccer, Academy impact, Athlete development 


\section{Introduction}

In recent years, the emphasis on identifying and developing young talented athletes in the pursuit of sporting excellence has led to national governing bodies and professional clubs investing considerable resources into Talent Identification and Development Systems (TIDS; Cobley, Schorer, \& Baker, 2012; Rongen, McKenna, Cobley, \& Till, 2018; Williams \& Reilly, 2000). Youth soccer academies are among the most popular TIDS in the UK, with approximately 10,000 youth players involved at any given point (Green, 2009). In 2012, the Premier League introduced the Elite Player Performance Plan (EPPP; The Premier League, 2011), aimingto increase the number of 'home-grown' players in the league by enhancing coaching quality and increasing contact time (Chesterton \& Wijnbergen, 2018) while also explicitly focusing upon player health, welfare and holistic development (Roe \& Parker, 2016). The increased focus on health, welfare and player development aligns with the ideas expressed by Williams \& Reilly (2000), who stated "the pursuit of excellence should not occur at the expense of the child's physical, emotional health, growth and development" (p.664665). Yet, contemporary narratives suggest tensions in simultaneously achieving both aims (Conn, 2017).

With reference to soccer academies, these tensions have been acknowledged by an upsurge in research exploring the impact of involvement on several physical outcomes. Recent studies have focussed on fitness development (Hammami et al., 2013), overtraining (Brink, Visscher, Coutts, \& Lemmink, 2012), injury and illness (e.g., Bowen, Gross, Gimpel, \& Li, 2016; Brink et al., 2010; Read et al., 2018; Tears et al., 2018), sleep quality and physical fatigue (Noon, James, Clarke, Akubat, \& Thake, 2015). However, given the growing emphasis on holistic player development (EPPP, 2011; Roe \& Parker, 2016) there is a need to look beyond the physical by also 
examining the impact of soccer academy involvement from a psychosocial perspective (Chin, McGregor, \& Daley, 2018; Rongen et al., 2018).

Here it is useful to outline the three elements that form the conceptual framework for this study. First, talented youth athletes, just like their age-group peers, need to achieve numerous developmental tasks including -but not limited toestablishing greater autonomy and detachment from parents; forming meaningful friendships and peer affiliation; successfully forming a rounded identity; formulating a positive self-concept, and developing a sense of future career direction (e.g., Kipp, 2017; Tamminen \& Braun, 2017). As a developmental period, adolescence covers a wide range of markers that signal maturation and healthy psychosocial development. TIDS and academies play host to these while trying to accelerate athletic performance development.

Second, TIDS and soccer academies can be characterised by several established practices and systemic features (Cobley, 2016). First, TIDS involve intensified and specialised training regimes which introduce high training frequency, training volumes and, year-round competition with fewer opportunities for rest (Bergeron et al., 2015). Second, TIDS are considered to be both prescriptive and restrictive (Beckmann, Elbe, Szymanski, \& Ehrlenspiel, 2006; Elbe, Szymanski, \& Beckmann, 2005), requiring athletes to adhere to highly structured full-time schedules, prioritise their sport over other activities (including education and spending time with friends) and be exposed to an environment dominated by adult-based concepts, values and extensive supervision (Bailey \& Collins, 2013; Diehl et al., 2012; Hendriksen, 2010). In some contexts, TIDS may endorse and embody the 'performance narrative', reinforcing a sole focus on, and an unwavering dedication towards, 'being an athlete'(Christensen \& Sorensen, 2009; Douglas \& Carless, 2006; McGillivray, Fearn, \& McIntosh, 2005). As a result, TIDS, 
including soccer academies, can be considered pressurised performance environments where performance is consistently scrutinised (Cushion \& Jones, 2006; Reeves, Nicholls \& McKenna, 2006), there is continuous competition for scarce positions (including contracts); and an ever-present threat of de-selection (Brown \& Potrac, 2009; Güllich, 2014). Such contexts can create an overly challenging, competitive, ego-based psychological and social climate for youth (Carless \& Douglas, 2013; Coakley, 1992; Cobley, 2016; Gould, 1993; Pankhurst \& Collins, 2013; Sabato, Walch, \& Caine, 2016).

Third, TIDS, and soccer academies are complex, open, dynamic systems in which young maturing athletes interact with these programmatic features likely producing a multitude of outcomes (Lerner, 2004; Philips et al., 2010; Rongen et al., 2018). Given these features TIDS may pose challenges for the achievement of typical adolescent development tasks and impose specific risks to healthy psychosocial development. Indeed, UK policy (Grey-Thompson, 2017), popular media accounts (Calvin, 2018), the International Olympic Committee (Bergeron et al., 2015) and academics (e.g. Wiersma, 2000; Sabato et al., 2016) have all raised concerns regarding the psychosocial outcomes of TIDS and specifically soccer academy involvement. Numerous theories offer frameworks that can and have been used to understand how and/or why the social context (such as sport and TIDS) impacts upon psychosocial development and well-being. Amongst others, existing literature has drawn on selfdetermination theory (Ryan \& Deci, 2000), identity theory (Erikson, 1968), theories of stress and coping (Lazarus \& Folkman, 1984; Nichols \& Polman, 2007), and theories of thriving (Brown et al., 2018). However, no single theory or framework has fully captured the multitude of aspects encapsulated by healthy psychosocial development. As such, we advocate the need to examine impact in a way that brings together the 
different aspects of psychosocial development to help provide a more comprehensive picture. To do so, the current study draws on program evaluation principles (Weiss, 1999). This approach advocates establishing a 'program theory' by outlining program features and their hypothesised associated outcomes, so that these can form the foundations of the evaluation process. Therefore, in the following paragraphs existing literature is used to mapwhich psychosocial impacts are commonly associated with TIDS programmatic and systemic features..

Psychosocially, relating back to the key established TIDS practices described earlier, four program features need to be considered. First, the feature of increased intensity and specialisation of training has been related to fatigue, compromised physical well-being (Bergeron et al., 2015; DiFiori et al., 2014; Malina, 2010; Wiersma, 2000), high perceived pressure and limited opportunities for recovery (Bergeron et al., 2015; DiFiori et al., 2014; Sabato et al., 2016). Second, the feature of prescriptive and demanding schedules combined with a sole focus on and unwavering dedication towards 'being an athlete' (e.g., soccer player) has been associated with a reduced sense of autonomy (Beckmann et al., 2006), social sacrifice (e.g., Beckmann et al. 2006; Knowles, Gastin \& Kremer, 2017; Miller \& Kerr, 2002) and the development of maladaptive, exclusive and strong athletic identities (e.g., Miller \& Kerr, 2002; Mitchell et al., 2014). Further, studies have reported difficulty in combining school and sport demands, resulting in reduced wellbeing and educational engagement (e.g., Miller \& Kerr, 2002; Cosh \& Tully, 2014; Christensen \& Sörensen, 2009). Third, the feature of continuous scrutiny and a pressurised performance environment can place athletes at risk of compromised psychological well-being, lowered moods, lowered sense of competence, and high perceived stress and the potential of ultimately burning out (e.g., Bergeron et al., 2015; DiFiori et al., 2014; Sabato et al., 2016; Sagar, Busch \& Jowett, 
2010).. The fourth feature of competition for scarce position availability may further exacerbate already lowered feelings of relatedness and belonging (Cushion \& Jones, 2006; Sagar et al., 2010).

While several studies have examined psychosocial outcomes of soccer academy involvement (e.g., burnout - Hill, Hall, Appleton, \& Kozub, 2008; impact on education Christensen \& Sörensen, 2009; mental health and depression - Jensen, Ivarsson, Fallby, Dankers, \& Elbe, 2018; identity development -Mitchell et al., 2014; developmental experiences and social exclusion -Taylor \& Bruner, 2012), all were based on crosssectional research designs. Although insightful, these studies only provide 'snapshots' as to the impact of TID involvement; they cannot provide the full picture of the stability and time course of these impacts. Only longitudinal research designs can achieve such insights (Cobley \& Till, 2017).

To our knowledge, six studies have explored psychosocial impacts of academy involvement longitudinally. Findings are equivocal with an almost 50/50 split in terms of reporting positive and negative outcomes over the course of one or more seasons. For example, three studies focusing on soccer academy involvement identified high and stable levels of autonomy, competence and relatedness (Adie, Duda \& Ntoumanis, 2010; 2012; Cheval, Chalabaev, Quested, Courvoisier, \& Sarrazin, 2017), self-esteem (Adie et al., 2010; Cheval et al., 2017), postive affect (Adie et al., 2010) and subjective vitality (Adie et al., 2012; Cheval et al., 2017). These studies also reported low and stable levels of negative affect (Adie et al, 2010), emotional and physical exhaustion (Adie et al., 2012) and burnout (Cheval et al., 2017). In contrast, the three other soccer academy specific studies reported decreases in wellbeing (motivation to train, sleep and recovery quality, appetite, soreness, fatigue and stress; Noon et al., 2015), accumulated stress and lack of recovery (Faude, Kellmann, Ammann, Schnittker, \& Meyer, 2011), 
decreasing levels of autonomy, competence and relatedness and increasing levels of burnout (Balaguer et al., 2012).

Unfortunately, these studies also had methodological limitations. Often they focused on specific, and arguably mostly psychological (i.e., mental and emotional) impacts, such as perceived stress, recovery and fatigue (Faude et al., 2011; Noon et al., 2015), vitality and exhaustion (Balaguer et al., 2012; Adie et al., 2012) and affective state and self-perception (Adie et al., 2010; Balaguer et al., 2012; Ivarsson et al., 2015). While these studies align to some of the commonly raised concerns around academy involvement (i.e., high perceived stress, lack of energy and fatigue, lowered mood, reduced sense of autonomy and competence) few have addressed socially focused concerns (i.e., restricted identity development, reduced sense of connection and time with friends, lack of autonomy away from the sport context, impact on life at school). Further, while each study has targeted one or two specific concerns, none provide a multidimensional overview of the different psychosocial impacts within the same sample. Doing so would establish a more comprehensive understanding.

Finally, as experimental or at least quasi-experimental research designs (i.e., with a comparison group) have not been used, existing studies are unable to answer whether academy exposure led to distinctive (un)healthy outcomes compared to other youth activities (e.g., school level soccer). To date only two studies deployed such a design focusing on the psychosocial impact of TIDS; Beckmann et al., (2006) and Knowles et al., (2017). Compared to non-TIDS involved youth, talented athletes reported similar load and recovery balance; better sport competence, social self-concept and social (peer and parent) relationships (Beckmann et al., 2006); as well as better health and wellbeing and low levels of burnout (Knowles et al., 2017). However, each study focused on the impact of dedicated sport schools, aiming to provide talented 
youth athletes with a well-balanced environment that combines education with training, either in Germany (Beckmann et al., 2006) or Australia (Knowles et al., 2017). Based on the limited and conflicting prior research related to the psychosocial impact of soccer academy involvement, alongside research describing the pressurised, challenging working culture in youth academies (e.g., Cushion \& Jones, 2006; Christensen \& Sörensen, 2009), the question remains whether soccer academy involvement can secure similarly positive psychosocial outcomes as those produced by dedicated sport schools.

Therefore, the purpose of the present study was to longitudinally evaluate the psychosocial impact of youth elite academy soccer involvement by comparing players to an age-matched soccer-active school sample. To provide a comprehensive assessment, this study included a variety of psychosocial measures deliberately targeting the most commonly raised concerns regarding soccer academy involvement (e.g., Bergeron et al., 2015; Sabato et al., 2016; Wiersma, 2000). While the evidence regarding the psychosocial impact of TIDS involvement is fairly equivocal, a number of well-reasoned arguments regarding the risks of TIDS involvement have been made (Grey-Thompson, 2017; Calvin, 2018; Bergeron et al., 2015;Wiersma, 2000; Sabato et al., 2016), these need to be robustly examined. Therefore, we hypothesised that academy players will report more negative psychosocial outcomes compared to their age-group peers. Further, based on the prevalence of varied and multifactorial stresses within TIDS it is hypothesised that psychosocial outcomes within academies may be less stable over time.

\section{Methods}

\section{Study Design}

A prospective longitudinal cohort design was employed to track youth elite soccer players and age-matched soccer-active school pupils over 12 months. Data collection 
occurred over one year in four equally spaced data collection periods $(\mathrm{T} 1=$ October, $\mathrm{T} 2$ $=$ February, $\mathrm{T} 3=$ May, $\mathrm{T} 4=$ September). All procedures received institutional ethics approval with informed assent and parental written consent obtained.

\section{Participants}

Fifty-eight male youth academy soccer players (12.98 \pm 1.61 years; under 12-under 16 age groups, weekly training time $=11.37 \pm 1.67$ hours; weekly competition time $=$ $1.28 \pm 0.26$ hours) and fifty-seven male age-matched soccer-active (but not involved in an academy of any standing) school pupils $(13.09 \pm 1.29$ years; weekly training time $=$ $2.06 \pm 1.56$ hours; weekly competition time $=1.61 \pm 1.14$ hours) participated in the study. The youth academy soccer players were sampled from one Category 1 (deemed to represent the optimum development model; EPPP, 2011) academy linked to a Premier League Football Club. The age-matched soccer-active school pupils were sampled from a secondary school within the North of England with an Ofsted rating of 2 (good). Given the potential links between socio-demographic status and psychosocial outcomes, it was deemed important to assess how similar both groups were in terms of sociodemographic status, especially as the two groups were sampled from geographically different locations in the North of England. To do so, participant postcodes were analysed for index of multiple deprivation, using a 1 (most deprived) to 32,844 (least deprived) ranking for England (Department for Communities and Local Government, 2015). The sample analysis showed similar distributions; $48 \%$ of academy players and $51 \%$ of school pupils were from areas amongst the $40 \%$ most deprived small areas in the country while $46 \%$ of academy players and $43 \%$ of school pupils were from areas amongst the $40 \%$ least deprived areas in the country. 


\section{Measures}

Data collection involved participants completing a booklet of 4 validated questionnaires that provided a multidimensional picture of psychosocial impact. Questionnaires were specifically selected to target key concerns regarding psychosocial outcomes outlined in the literature. Table 1 presents an overview of how questionnaires mapped to identified concerns.

$* * * *$ Table 1 near here****

\section{Recovery-Stress Questionnaire for Athletes (RESTQ-Sport)}

The perceived level of stressors and recovery activities was assessed with the RESTQSport (Kellmann \& Kallus, 2001). In line with recommendations for longitudinal research (Kellmann, Altenburg, Lormes, \& Steinacker, 2001), the 52-item version was used. Participants were asked to answer items with reference to the previous week on a 7 - point Likert-type scale $(0=$ never, $1=$ rarely, $2=$ sometimes, $3=$ often, $4=$ more often, 5 = very often, 6 = always). The RESTQ-Sport consists of twelve general and seven sport-specific scales. In addition, these scales can be summarised in higher order scales to provide general stress ( $\Sigma 7$ general stress subscales), sport-specific stress $(\Sigma 3$ sport-specific stress subscales), general recovery ( $\Sigma 5$ general recovery subscales) and sport-specific recovery scores ( $\Sigma 4$ sport-specific recovery subscales), with higher scores indicating higher stress and higher recovery. The RESTQ-52 sport has good construct validity and good-to-satisfactory internal reliability (i.e., subscales' $\alpha$ 's ranged from 0.54 - 0.92; Kellmann \& Kallus, 2001).

2. Balanced Measure of Psychological Needs (BMPN). 
The BMPN (Sheldon \& Hilpert, 2012) assessed the extent to which participants perceive their needs for autonomy, competence and relatedness to be met. As previous literature has highlighted the importance of treating the degree to which needs are satisfied and thwarted (i.e., actively impeded) as two separate constructs (e.g., Cheval et al., 2017; Cordeiro, Paixão, Lens, Lacante, \& Sheldon, 2016), the BMPN consists of 18 items assessing both the satisfaction and thwarting of basic psychological needs. The questionnaire stem was 'Below are some statements about feelings and thoughts, please circle the number that best describes how true each statement was for you in the last week'. To allow for comparison between pupils and academy players, the assessment of need satisfaction and thwarting targeted the general level (i.e. how they felt overall) rather than context specific (i.e. how they felt at school or at the academy). Participants were asked to rate items on a 1 (not at all true), 3 (somewhat true) to 5 (very true) scale. Six subscale scores were calculated by averaging the three items scores per scale; subscale scoreswere taken forward into analysis. Sound psychometric quality with good construct validity and adequate reliability has been reported for the BMPN ( $\alpha$ ranging from 0.69 - 0.85 for the three-item subscales; Sheldon \& Hilpert, 2012).

\section{KIDSCREEN-27}

To assess perceptions of physical, psychological and social well-being the KIDSCREEN-27 (The KIDSCREEN Group Europe, 2006) was used. The KIDSCREEN-27 assesses five dimensions of Health-Related Quality of Life (HRQoL) covering (i) physical well-being, (ii) psychological well-being, (iii) autonomy and parent relationships, (iv) social support and peers and (v) school environment. This 27 items questionnaire is suitable for use with children and adolescents aged 8-18 years (The KIDSCREEN Group Europe, 2006). Participants were asked to answer items with 
reference to the previous week on a 5-point Likert-type scale with three different sets of responses: a) poor, fair, good, very good, excellent ; b) not at all, slightly, moderately, very, extremely ; c) never, seldom, quite often, very often, always. Four negatively formulated items were re-coded before calculating subscale scores. Raw subscale scores were converted to Rasch person parameter estimates, into z-values and then into Tvalues, using the SPSS syntax provided. This transformation results in subscale scores with a scale mean around 50 and standard deviation around 10, with higher values indicating. higher HRQoL (The Kidscreen Group Europe, 2006). Transformed subscale scores were taken forward into the analysis. All KIDSCREEN-27 domains have reported satisfactory-to-good internal consistency ( $\alpha=0.79$ to 0.84$)$ and two-week testreliability was deemed adequate for all dimensions with ICC's ranging from 0.61-0.74 (the KIDSCREEN group Europe, 2006).

\section{Athletic Identity Measurement Scale (AIMS)}

To examine concerns around athletic identity, participants' perception of their identity in relation to sport was assessed using the seven-item AIMS (Brewer \& Cornelius, 2001). Each item is rated on a Likert scale ranging from 1 (strongly disagree) to 7 (strongly agree). For the unidimensional scale, the seven items are summed; higher scores represent a stronger athletic identity. The AIMS can simultaneously be treated as multidimensional, with three subscales, exclusivity ( 2 items), negative affectivity ( 2 items), and social identity (3 items). Subscale scores are calculated as the mean of each scale's respective items, resulting in means between 1-7. High 'exclusivity' scores indicate self-worth is strongly linked to the athlete role, high 'negative affectivity' scores indicate more negative emotions as a result of unwanted sporting outcomes and high 'social identity' scores indicate a strong sense of occupying the role of an athlete. 
Both the unidimensional scale and the three subscales are used in subsequent analysis. Support for the validity and reliability of the 7-item AIMS was provided by its authors ( $\alpha=0.81$ for unidimensional scale, Brewer \& Cornelius, 2001). More recent studies reported additional psychometric support for three first-order factors sub-ordinate to one higher-order athletic identity factor (Tasiernski et al., 2004; Visek et al., 2008).

\section{Statistical analysis}

Only complete cases (i.e., completed measures at all four time points) were analysed. To ensure complete case analysis was appropriate and to establish no complete case bias was present, dropout analysis was performed (Osborne, 2013). This revealed no differences in the main study variables at $\mathrm{T} 1(\mathrm{p}>0.05)$ between dropouts and players/pupils who participated in the four data-collection time points.. Complete data from 77 players $(n=33 ; 58.6 \%)$ and school pupils $(n=44 ; 85.2 \%)$ were included in the final analysis. Descriptive statistics (mean, SD, range, skewness and kurtosis) were calculated for all dependent variables. Scale reliability was assessed using Cronbach's alpha. Convention stipulates that an $a<0.50$, signifies poor reliability and $a>0.70$ signifies good reliability (Cortina, 1993), although $a>0.50$ is acceptable for group comparisons (Hoener \& Roth, 2002). For the RESTQ-Sport, 7 of the 19 subscales showed poor internal consistency $(a<0.50)$ at two or more timepoints. The four overarching scales showed good-to-excellent internal reliability across all four time points $(a=0.71-0.91)$. As such, the choice was made to only include the four higher order subscales in subsequent analysis. Across all other measures, all subscales, bar competence dissatisfaction showed acceptable internal consistency $(a>0.50)$ for data obtained on at least 3 of the 4 time points. Low alphas can be caused by scales consisting of few items and in this case, scales can still be usefully included in analysis 
(Cortina, 1993), as long as the low alpha value is discussed as a limitation (Schmitt, 1996). The competence thwarting scale is made up of only 3 items which may explain its low alpha. With this in mind all scales were included in the analysis, although caution may be warranted in interpreting the competence dissatisfaction subscale. For all scales taken forward into analysis, the reliabilities at each time point can be found in the supplementary table.

Assumptions of repeated measures multivariate analyses were checked through inspecting the normality of the distribution of the difference scores (from T1 through to T4) and graphically via P-P plots (Maxwell \& Delaney, 2004). To examine the impact of soccer vs. school pupil on levels and changes in psychosocial outcomes over the course of a year, five independent mixed between-within repeated measures multivariate analysis of variance (MANOVAs) were conducted. Where Mauchley's test of sphericity was violated, degrees of freedom were adjusted using the GreenhouseGeiser correction (Field, 2009). To minimise the likelihood of Type-1 errors, a Bonferroni corrected alpha level of 0.01 was used. Effect sizes were examined using partial eta squared $(\eta \mathrm{p} 2)$, and adopting the following criteria: $0.01=$ small, $0.06=$ medium, 0.138 = large (Pallant, 2013; Tabachnick \& Fidell, 2007). All analysis was conducted using SPSS V 22.0.

\section{Results}

The mean \pm standard deviation all variables at all four time points (T1 $-\mathrm{T} 4)$ are presented in Table 2.

$$
\text { ****Table } 2 \text { near here } * * * *
$$

\section{Stress and Recovery}


The repeated measures MANOVA, showed no significant group and time interaction ( $\mathrm{p}$ $=0.24, \mathrm{ES}=$ small, $\left.\eta_{p}{ }^{2}=0.02\right)$ or main group effect $\left(\mathrm{p}=0.04 ; \mathrm{ES}=\right.$ medium, $\left.\eta_{p}{ }^{2}=0.13\right)$ for any of the subscales of the REST-Q questionnaire, demonstrating that there were no differences between soccer players and school pupils. However, a significant main effect of time was present $\left(\mathrm{p}<0.01, \mathrm{ES}=\right.$ medium, $\left.\eta_{p}{ }^{2}=0.06\right)$. Univariate analysis showed general $\left(\mathrm{p}<0.01 ; \mathrm{ES}=\right.$ medium, $\left.\eta_{p}{ }^{2}=0.07\right)$ and sport specific stress $(\mathrm{p}<0.01$; $\mathrm{ES}=$ medium, $\left.\eta_{p}{ }^{2}=0.07\right)$ decreasing over time and general $\left(\mathrm{p}<0.01 ; \mathrm{ES}=\right.$ medium, $\eta_{p}{ }^{2}$ $=0.06)$ and sport specific recovery $\left(\mathrm{p}<0.01 ; \mathrm{ES}=\right.$ medium,$\left.\eta_{p}{ }^{2}=0.07\right)$ improving over time irrespective of group. These findings are further supported by pairwise comparisons, which revealed a reduction in general ( $\mathrm{T} 1$ to $\mathrm{T} 3, \mathrm{p}<0.01)$ and sportspecific $(\mathrm{T} 1$ to $\mathrm{T} 3, \mathrm{p}=0.018 ; \mathrm{T} 1$ to $\mathrm{T} 4, \mathrm{p}=0.01)$ stress and an increase in general $(\mathrm{T} 1$ to $\mathrm{T} 4, \mathrm{p}=0.04)$ and sport specific $(\mathrm{T} 1$ to $\mathrm{T} 4, \mathrm{p}<0.01)$ recovery for both groups across the time periods.

$* * * *$ Figure 1 near here $* * * *$

\section{Autonomy, competence and relatedness}

Repeated measures MANOVA identified no significant group and time interaction $(\mathrm{p}=$ $\left.0.61, \eta_{p}^{2}=0.02\right)$, nor significant main effects of time $\left(\mathrm{p}=0.03, \eta_{p}{ }^{2}=0.05\right)$ or group $(\mathrm{p}=$ $0.05, \eta_{p}{ }^{2}=0.16$ ), indicating no significant differences between groups or across time for the need satisfaction and thwarting questionnaire.

\section{Health-related quality of life}

For the health-related quality of life questionnaire, repeated measures MANOVA identified a significant interaction between group and time $\left(\mathrm{p}<0.01, \mathrm{ES}=\right.$ small, $\eta_{p}{ }^{2}=$ 
$0.05)$, but no significant main effects of group $\left(\mathrm{p}=0.05 ; \mathrm{ES}=\operatorname{large}, \eta_{p}{ }^{2}=0.14\right)$ or time $\left(\mathrm{p}=0.08 ; \mathrm{ES}=\right.$ small, $\left.\eta_{p}{ }^{2}=0.03\right)$. Univariate analysis for the health related quality of life questionnaire revealed a difference how academy players and school pupils perceived their quality of life within the school environment over time, indicated by a significant interaction effect for the HRQoL school dimension $(\mathrm{p}<0.01, \mathrm{ES}=$ medium, $\eta_{p}{ }^{2}=0.06$ ), see Figure 2. Academy players and school pupils reported similar school related quality of life from $\mathrm{T} 1$ to $\mathrm{T} 3$, with a significant difference in scores at $\mathrm{T} 4$ ( $\mathrm{p}<$ $0.01, \mathrm{ES}=$ medium,$\left.\eta_{p}{ }^{2}=0.09\right)$, as a result of an increase in school related quality of life for school pupils during this period. No other significant differences were observed between groups for health-related quality of life categories.

$* * * *$ Figure 2 near here $* * * *$

\section{Athletic identity}

For athletic identity assessed as a unidimensional construct (i.e. total athletic identity), repeated measures MANOVA identified no significant group and time interaction $\left(\mathrm{p}=0.74 ; \mathrm{ES}=\right.$ small, $\left.\eta_{p}{ }^{2}=0.02\right)$, nor a significant main effect of time $(\mathrm{p}=0.08 ; \mathrm{ES}=$ medium, $\left.\eta_{p}{ }^{2}=0.08\right)$. However, total athletic identity was higher for academy players in comparison to school pupils, as revealed by a significant main effect for group $(\mathrm{p}<0.01$; $\mathrm{ES}=$ large,$\left.\eta_{p}{ }^{2}=0.16\right)$,). Post-hoc analyses exploring the simple main effects, indicated that academy players outscored school pupils in terms 'total athletic identity' scores at each time point (T1-T4, all $\mathrm{p} \leq 0.01)$.

For athletic identity assessed as a multidimensional construct, repeated measures MANOVA identified no significant group and time interaction $\left(\mathrm{p}=0.87 ; \mathrm{ES}=\right.$ small, $\eta_{p}{ }^{2}$ $=0.01)$, nor a significant main effect of time $\left(\mathrm{p}=0.50 ; \mathrm{ES}=\right.$ small, $\left.\eta_{p}{ }^{2}=0.01\right)$. However, athletic identity was higher for academy players in comparison to school 
pupils at all time points, as revealed by a repeated measures MANOVA significant main effect for group $\left(\mathrm{p}<0.01 ; \mathrm{ES}=\right.$ large,$\left.\eta_{p}{ }^{2}=0.26\right)$. This difference was the result of soccer players reporting higher 'Exclusivity' scores ( $\mathrm{p}<0.01$; ES $=$ large, $\left.\eta_{p}{ }^{2}=0.25\right)$. Post-hoc analyses exploring the simple main effects, indicated that academy players outscored school pupils in terms of 'exclusivity' scores at each time point (T1-T4, all p $\leq 0.01)$. No differences were observed in the Social identity, or Negative affectivity subscales (Figure 3)

****Figure 3 near here $* * * *$

\section{Discussion}

This study aimed to longitudinally evaluate the psychosocial outcomes of academy involvement and is the first to compare academy players with age-matched, though still soccer-active, counterparts. As such, this study provides important new evidence, beyond that established from cross-sectional studies and simple pre-post designs that have prevailed in this field. Recruiting a soccer-active pupil comparison group permitted comparisons of psychosocial outcomes relative to 'normal' adolescents. To enhance relevance to contemporary concerns for academy players, we deliberately targeted the most commonly raised psychosocial issues identified in literature.

\section{Predominantly positive psychosocial impacts}

The findings revealed that across a 12-month period youth soccer players reported largely stable and positive psychosocial outcomes. Importantly, on almost all measures academy players reported similar scores to those of age-matched soccer-active school pupils. While previous soccer specific research (Adie et al., 2010, 2012; Balaguer et al., 2012; Cheval et al., 2017; Faude et al., 2011; Ivarsson et al., 2015; Noon et al., 2015) 
provided initial insight into psychosocial outcomes and how they change over time, few studies had addressed how these patterns compare to age matched counterparts. In contrast to our hypotheses, and despite prominent concerns around the potential negative impact of TIDS (e.g., DiFiori et al., 2014; Malina, 2010; Sabato et al., 2016), our findings indicate that academy involvement was not necessarily associated with, nor did it induce over time, negative psychosocial outcomes. Rather, academy players reported positive trends related to increased opportunities to recover and decreased perceived stress, signifying potentially appropriate load-recovery balance in the examined context.

Players reported that their basic psychological needs for autonomy, competence and relatedness were often satisfied and they were rarely dissatisfied. Further, players consistently experienced good health-related quality of life in terms of physical wellbeing, psychological well-being, autonomy, parent and peer relationships and in their school environment. While school-related quality of life remained stable for academy players, school pupils reported an improvement from T3 - T4. Given the pattern of the other findings, and as academy players scores were comparable to normative data for European males of the same age (the KIDSCREEN Group Europe, 2006), these findings are more likely explained by positive changes within the school environment and should not be interpreted as negative effects from academy involvement.

Findings in this current study both support (Adie et al., 2010, 2012; Brink et al., 2010; Cheval et al., 2017; Ivarsson et al., 2015) and contradict (Balaguer et al., 2012; Faude et al., 2011; Noon et al., 2015) previous studies examining load-recovery balance, need satisfaction, feeling energised and affective states in youth soccer players. Similar to Adie and colleagues (2012), findings suggested an improvement of loadrecovery balance over time, suggesting players became better at dealing with the 
demands of soccer academy involvement. Given that a poor load-recovery balance has been linked to injury, illness (Brink et al., 2010; Brink et al., 2012; Gabbett, Whyte, Hartwig, Wescombe, \& Naughton, 2014) and burnout (Bergeron et al., 2015; DiFiori et al., 2014), these are promising findings. They give reasons to be optimistic, rather than pessimistic about the current academy experience and point to the capability of practitioners and soccer academy environments to preserve and facilitate positive psychosocial outcomes. The high and stable levels of need satisfaction are also encouraging given their positive links to athlete wellbeing, thriving and positive developmental outcomes (Adie, Duda, \& Ntoumanis, 2008; Lundqvist \& Raglin, 2015; Ryan \& Deci, 2000). Further, as one of the first studies to separately assess need satisfaction and need thwarting in youth soccer players, our findings regarding low and stable need thwarting are particularly positive, as 'psychological need thwarting' has been highlighted as an important predictor for ill-being (Cheval et al., 2017).

\section{Athletic identity as a risk}

Notwithstanding positive findings, academy players reported stable and consistently higher total and exclusive athletic identity relative to the soccer-active school pupils. While identifying with being an athlete is associated with psychological benefits and values (e.g., self-esteem, positive body image, work ethic), an exaggeratedly narrow one-dimensional (strong and exclusive) identity has been associated with a host of negative consequences in the short- and long-term, including;; i) inadequate coping with (inevitable) setbacks, e.g. injury, deselection (Brown \& Potrac, 2009; Stambulova, 2003); (ii) overtraining/burnout (Coakley, 1992); (iii) willingness to self-sacrifice or risk one's health (Miller \& Hoffman, 2009; Schnell, Mayer, Diehl, Zipfel, \& Thiel, 2014); (iv) identity-foreclosure, a lack of focus on education, and delayed career development (Miller \& Kerr, 2003; Murphy, Petitpas, \& Brewer, 1996), and (v) being 
ill-prepared for transitions out of sport (e.g., Park, Lavallee, \& Tod, 2013; Pummell, Harwood, \& Lavallee, 2008) resulting in identity loss, depression and jeopardized wellbeing (Douglas \& Carless, 2006, 2009).

The athletic identity scores reported by our sample are comparable to those reported in other adolescent samples, including soccer players (Mitchell, et al., 2014) and talented youth athletes across a variety of sports (Verkooijen van Hove, \& Dik, 2012). Collectively, these findings suggest that intensive engagement with elite youth sport may place youth athletes at risk for developing an - unhealthily strong and exclusive - athletic identity. Importantly, though, despite reporting a strong athletic identity, our sample did not show any further current indicators of negative psychosocial impact.

Reviewing the host of largely positive findings regarding psychosocial outcomes from the current study, these are likely to reflect the quality of programme management and climate of the examined academy.. Contemporary research highlights that the impact of academy (or TIDS) involvement is neither good nor bad per se, instead, attention should be given to how specific programmes are designed, implemented and managed (Rongen et al., 2018). Indeed, the two studies (Beckmann et al., 2006; Knowles et al., 2017) reporting favourable psychosocial outcomes for talented athletes compared to a group on 'normal' school pupils were situated within dedicated sport schools, deliberately designed to provide an optimised and well-balanced environment for youth elite athletes. Similarly, recent research suggests the importance of environmental qualities, such as how well coaches understand and support athletes holistically, the quality of coach-athlete relationships, as well as perceptions of autonomy-supportive versus controlling social environments (Appleton \& Duda, 2016; Gucciardi, Stamatis, \& Ntoumanis, 2017; Ivarsson et al., 2015). Future research would 
benefit from exploring not only if, but also how, academy environments can sustain and promote positive psychosocial outcomes.

\section{Limitations}

Although this study represents a first attempt to explore the psychosocial outcomes of academy involvement using a quasi-experimental longitudinal design, it is not without limitations. First, participants were from one soccer academy and school context, and findings may not be generalised across the sport or to other TIDS. Future research should replicate such comparative longitudinal research designs across a range of contexts, including both male and female talented athletes to establish whether, and under what circumstances, such findings are replicated or differ. Second, this study did not address inter-individual differences in psychosocial outcomes. Future research could deploy longitudinal case-study approaches to track the development of a smaller sample of youth players in depth (e.g., Cobley, Till, O’Hara, Cooke, \& Chapman, 2014), or examine larger sample sizes using different statistical techniques such as multilevel modelling, linear mixed modelling or latent growth models (e.g., Adie, et al., 2012; Cheval, et al., 2017; Ivarsson, et al., 2015).

\section{Conclusion}

The current study provides robust evidence suggesting that elite youth soccer academy contexts can cater for athletes' psychological needs, provide an appropriate training/competitive load-recovery balance (linked to not feeling over-trained or burntout) and can keep stable and facilitate a sense of positive physical, psychological and social wellbeing. Nonetheless, the presence of strong and exclusive athletic identities relative to age-matched school pupils represents a potential risk factor for the healthy development of the players involved. As concerns around the potential negative impact 
prevail, the present results should encourage academy staff that they can potentially facilitate positive psychosocial outcomes, while remaining vigilant and actively monitoring other facets (e.g., athletic identity) which may lead to problems in particular circumstances.

\section{References}

Adie, J. W., Duda, J., \& Ntoumanis, N. (2008). Achievement goals, competition appraisals, and the psychological and emotional welfare of sport participants. Journal of Sport \& Exercise Psychology, 30 (3), 302-322.

Adie, J. W., Duda, J. L., \& Ntoumanis, N. (2010). Achievement goals, competition appraisals, and the well- and ill-being of elite youth soccer players over two competitive seasons. Journal of Sport and Exercise Psycholy, 32(4), 555-579.

Adie, J. W., Duda, J. L., \& Ntoumanis, N. (2012). Perceived coach-autonomy support, basic need satisfaction and the well- and ill-being of elite youth soccer players: A longitudinal investigation. Psychology of Sport and Exercise, 13(1), 51-59.

Appleton, P. R., \& Duda, J. L. (2016). Examining the interactive effects of coachcreated empowering and disempowering climate dimensions on athletes' health and functioning. Psychology of Sport and Exercise, 26, 61-70.

Bailey, R., \& Collins, D. (2013). The Standard Model of Talent Development and Its Discontents. Kinesiology Review, 2(4), 248-259.

Balaguer, I., Gonzalez, L., Fabra, P., Castillo, I., Merce, J., \& Duda, J. L. (2012). Coaches' interpersonal style, basic psychological needs and the well- and illbeing of young soccer players: a longitudinal analysis. Journal of Sports Sciences, 30(15), 1619-1629.

Beckmann, J., Elbe, A., Szymanski, B., \& Ehrlenspiel, F. (2006). Chancen und risiken: Vom leben im verbundsystem von schule und leistungssport. Psychologische, soziologische und sportliche leistungsaspekte (Auflage 2006 ed.) Bonn: Bundesinstitut für Sportwissenschaft.

Bergeron, M. F., Mountjoy, M., Armstrong, N., Chia, M., Côté, J., Emery, C. A., . . Engebretsen, L. (2015). International Olympic Committee consensus statement on youth athletic development. British Journal of Sports Medicine, 49(13), 843851. 
Bowen, L., Gross, A. S., Gimpel, M., \& Li, F.-X. (2016). Accumulated workloads and the acute:chronic workload ratio relate to injury risk in elite youth football players. British Journal of Sports Medicine, 51, 452-459

Brewer, B., \& Cornelius, A. E. (2001). Norms and factorial invariance of the Athletic Identity Measurement Scale (AIMS). Academic Athletic Journal, 16, 103-113.

Brink, M. S., Visscher, C., Arends, S., Zwerver, J., Post, W. J., \& Lemmink, K. A. P. M. (2010). Monitoring stress and recovery: new insights for the prevention of injuries and illnesses in elite youth soccer players. British Journal of Sports Medicine, 44(11), 809-815.

Brink, M. S., Visscher, C., Coutts, A. J., \& Lemmink, K. A. P. M. (2012). Changes in perceived stress and recovery in overreached young elite soccer players. Scandinavian Journal of Medicine \& Science in Sports, 22(2), 285-292.

Brown, D. J., Arnold, R., Reid, T., \& Roberts, G. (2018). A qualitative exploration of thriving in elite sport. Journal of Applied Sport Psychology, 30(2), 129-149.

Brown, G., \& Potrac, P. (2009). 'You've not made the grade, son': De-selection and identity disruption in elite level youth football. Soccer \& Society, 10(2), 143159.

Calvin, M. (2018). No hunger in paradise. The players. The journey. The dream. London, UK. : Cornerstone.

Carless, D., \& Douglas, K. (2013). "In the boat" but "Selling myself short": Stories, narratives, and identity development in elite sport. The Sport Psychologist, 27(1), 27-39.

Cheval, B., Chalabaev, A., Quested, E., Courvoisier, D. S., \& Sarrazin, P. (2017). How perceived autonomy support and controlling coach behaviors are related to welland ill-being in elite soccer players: A within-person changes and betweenperson differences analysis. Psychology of Sport and Exercise, 28, 68-77.

Chin, W. O., McGregor, P., \& Daley, C. (2018). The boy behind the bravado: Player advanced safety and support in a professional football academy setting. Sport \& Exercise Psychology Review, 14(1), 65-79.

Christensen, M. K., \& Sörensen, J. K. (2009). Sport of School? Dreams and dilemmas for talented young Danish football players. European Physical Education Review, 15(1), 115-133. 
Coakley, J. (1992). Burnout among adolescent athletes: A personal failure or social problem? Sociology of Sport Journal, 9(3), 271-285.

Cobley, S. (2016). Talent Identification and Development in Youth Sport. In Ken Green, Andy Smith (Eds.), Routledge Handbook of Youth Sport, (pp. 476-491). Abingdon: Routledge.

Cobley, S., Schorer, J., \& Baker, J. (2012). Identification and development of sport talent: A brief introduction to a growing field of research and practice. In J. Baker, S. Cobley \& J. Schorer (Eds.), Talent identification and development in sport: International perspectives. London: Routledge.

Cobley, S., Till, K. (2017). Longitudinal studies of athlete development: Their importance, methods and future considerations. In Joseph Baker, Stephen Cobley, Jorg Schorer, Nick Wattie (Eds.), Routledge Handbook of Talent Identification and Development in Sport, (pp. 250-268). Abingdon: Routledge.

Cobley, S., Till, K., O’Hara, J., Cooke, C., \& Chapman, C. (2014). Variable and changing trajectories in youth athlete development: further verification in advocating a long-term inclusive tracking approach. Journal of Strength and Conditioning Research, 28(7), 1959-1970.

Cordeiro, P., Paixão, P., Lens, W., Lacante, M., \& Sheldon, K. (2016). Factor structure and dimensionality of the balanced measure of psychological needs among Portuguese high school students. Relations to well-being and ill-being. Learning and Individual Differences, 47, 51-60.

Cortina, J. M. (1993). What is coefficient alpha? An examination of theory and applications. Journal of Applied Psychology, 78(1), 98-104.

Cushion, C., \& Jones, R. L. (2006). Power, discourse, and symbolic violence in professional youth soccer: The case of Albion Football club. Sociology of Sport Journal(2), 142-161.

Department for Communities and Local Government (2015). The English Index of Multiple Deprivation (IMD) 2015 - Guidance.

Diehl, K., Thiel, A., Zipfel, S., Mayer, J., Litaker, D. G., \& Schneider, S. (2012). How healthy is the behavior of young athletes? A systematic literature review and meta-analyses. Journal of Sports Science \& Medicine, 11(2), 201-220.

DiFiori, J. P., Benjamin, H. J., Brenner, J. S., Gregory, A., Jayanthi, N., Landry, G. L., \& Luke, A. (2014). Overuse injuries and burnout in youth sports: a position 
statement from the American Medical Society for Sports Medicine. British Journal of Sports Medicine, 48(4), 1-15.

Douglas, K., \& Carless, D. (2006). Performance, discovery, and relational narratives among women professional tournament golfers. Women in Sport and Physical Activity Journal, 15 (2), 14-27.

Douglas, K., \& Carless, D. (2009). Abandoning the performance narrative: Two women's wtories of transition from professional sport. Journal of Applied Sport Psychology, 21(2), 213-230.

Elbe, A., Szymanski, B., \& Beckmann, J. (2005). The development of volition in young elite athletes. Psychology of Sport \& Exercise, 6, 559-569.

Erikson, E. H. (1968). Identity: Youth and crisis. New York: Norton.

Faude, O., Kellmann, M., Ammann, T., Schnittker, R., \& Meyer, T. (2011). Seasonal changes in stress indicators in high level football. International Journal of Sports Medicine, 32(4), 259-265.

Field, A. (2013) Discovering statistics using IBM SPSS statistics. London: Sage Gabbett, T., Whyte, D., Hartwig, T., Wescombe, H., \& Naughton, G. (2014). The Relationship Between Workloads, Physical Performance, Injury and Illness in Adolescent Male Football Players. Sports Medicine, 44(7), 989-1003.

Gould, D. (1993). Intensive sport participation and the prepubescent athlete: competitive stress and burnout. In B. R. Cahill \& A. J. Pearl (Eds.), Intensive participation in children's sports (pp. 19-38). Champaign, IL: Human Kinetics.

Green, C. (2009). Every boy's dream. English football future on the line. London: A\&C Publishers Ltd.

Grey-Thompson, T. (2017). Duty of Care in Sport Review: Independent Report to Government.

Gucciardi, D. F., Stamatis, A., \& Ntoumanis, N. (2017). Controlling coaching and athlete thriving in elite adolescent netballers: The buffering effect of athletes' mental toughness. Journal of Science and Medicine in Sport, 20(8), 718-722.

Güllich, A. (2014). Selection, de-selection and progression in German football talent promotion. European Journal of Sport Science, 14(6), 530-537.

Hammami, M. A., Ben Abderrahmane, A., Nebigh, A., Le Moal, E., Ben Ounis, O., Tabka, Z., \& Zouhal, H. (2013). Effects of a soccer season on anthropometric characteristics and physical fitness in elite young soccer players. Journal of Sports Sciences, 31(6), 589-596. 
Hendriksen, K. (2010). The ecology of talent development in sport: A multiple case study of succesful athletic talent development environments in Scandinavia (Doctoral dissertation). University of Southern Denmark.

Hill, A. P., Hall, H. K., Appleton, P. R., \& Kozub, S. A. (2008). Perfectionism and burnout in junior elite soccer players: The mediating influence of unconditional self-acceptance. Psychology of Sport and Exercise, 9(5), 630-644.

Hoener, O., \& Roth, K. (2002). Klassiche Testtheorie: Die Gütekriterien Sportwissenschaftlicher Erhebungsmethoden [Classical Test Theory: Test control criteria of survey methods in sport science]. In R. Singer \& K. Willimczik (Eds.), Sozialwissenschaftliche Forschungsmethoden in der Sportwissenschaft (pp. 29-54). Hamburg: Czwalina.

Ivarsson, A., Stenling, A., Fallby, J., Johnson, U., Borg, E., \& Johansson, G. (2015). The predictive ability of the talent development environment on youth elite football players' well-being: A person-centered approach. Psychology of Sport \& Exercise, 16, 15-23.

Jensen, S. N., Ivarsson, A., Fallby, J., Dankers, S., \& Elbe, A.-M. (2018). Depression in Danish and Swedish elite football players and its relation to perfectionism and anxiety. Psychology of Sport and Exercise, 36, 147-155.

Kellmann, M., Altenburg, D., Lormes, W., \& Steinacker, J. M. (2001). Assessing stress and recovery during preparation for the World Championships in rowing. / Evaluation du stress et de la recuperation pendant une preparation aux championnats du monde d ' aviron. Sport Psychologist, 15(2), 151-167.

Kellman, M. \& Kallus, K. (2001) Recovery-Stress Questionnaire for athletes. Champaign, IL: Human Kinetics.

Kipp, L.E. (2017). Development considerations for working with young athletes. In Knight, C. J., Harwood, C. G., \& Gould, D. (Eds.). Sport psychology for young athletes. New York: Routledge.

Knowles, O., Gastin, P. B., \& Kremer, P. (2017). Time use and health and wellbeing outcomes of sport school students in Australia. Sport Sciences for Health, 13(1), 427-435.

Lazarus, R. S., \& Folkman, S. (1984). Stress, appraisal, and coping. New York: Springer. 
Lerner, R. (2004). Diversity in individual - context relations as the basis for positive development across the lifespan: A developmental perspective for theory, research and application (The 2004 Society for the study of Human development presiential address). Research in Human Development, 1(4), 327-346.

Lundqvist, C., \& Raglin, J. S. (2015). The relationship of basic need satisfaction, motivational climate and personality to well-being and stress patterns among elite athletes : An explorative study. [Review]. Motivation and Emotion, 39(2), 237-246.

Malina, R. M. (2010). Early sport specialization: Roots, effectiveness, risks. Current Sports Medicine Reports, American College of Sports Medicine, 9(6), 364-371.

Maxwell, S. E., \& Delaney, H. D. (2004). Designing experiments and analyzing data: A model comparison perspective (2nd ed.) London: Lawrence Erlbaum Associates Publishers.

McGillivray, D., Fearn, R., \& McIntosh, A. (2005). Caught up in and by the beautiful game: A case study of Scottish professional footballers. Journal of Sport \& Social Issues, 29(1), 102-123.

Miller, K. E., \& Hoffman, J. H. (2009). Mental well-being and sport-related identities in college students. Sociology of Sport Journal, 26(2), 335-356.

Miller, P. S., \& Kerr, G. A. (2002). The Athletic, Academic and Social Experiences of Intercollegiate Student-Athletes. Journal of Sport Behavior, 25(4), 346-367 .

Miller, P. S., \& Kerr, G. A. (2003). The Role Experimentation of Intercollegiate Student Athletes. Sport Psychologist, 17(2), 196-219.

Mitchell, T. O., Nesti, M., Richardson, D., Midgley, A. W., Eubank, M., \& Littlewood, M. (2014). Exploring athletic identity in elite-level English youth football: a cross-sectional approach. Journal of Sports Sciences, 32(13), 1294-1299.

Murphy, G. M., Petitpas, A. J., \& Brewer, B. W. (1996). Identity foreclosure, athletic identity, and career maturity in intercollegiate athletes. The Sport Psychologist, 10(3), 239-246.

Nicholls, A., \& Polman, R. C. J. (2007). Stressors, coping, and coping effectiveness among players from the England under-18 rugby union team. Journal of Sport Behavior, 30, 199-218. 
Noon, M. R., James, R. S., Clarke, N. D., Akubat, I., \& Thake, C. D. (2015). Perceptions of well-being and physical performance in English elite youth footballers across a season. Journal of Sports Sciences, 33(20), 2106-2115.

Osborne, J. (2013). Dealing with missing or incomplete data: Debunking the myth of emptiness. In J. Osborn (Ed.), Best practices in data cleaning: A complete guide to everything you need to do before and after collecting your data. London: Sage Publications.

Pallant, J. (2005) SPSS Survival manual: a step by step guide to data analysis using SPSS. Australia: Allen \& Uwin.

Pankhurst, A., \& Collins, D. (2013). Talent Identification and Development: The Need for Coherence Between Research, System, and Process. Quest, 65(1), 83-97.

Park, S., Lavallee, D., \& Tod, D. (2013). Athletes' career transition out of sport: a systematic review. International Review of Sport and Exercise Psychology, 6(1), 22-53.

Philips, E., David, K., Renshaw, I. \& Portus, M. (2010). Expert performance in sport and the dynamics of talent development. Sports Medicine, 40(4), 271-283.

Pummell, B., Harwood, C., \& Lavallee, D. (2008). Jumping to the next level: A qualitative examination of within-career transition in adolescent event riders. Psychology of Sport and Exercise, 9(4), 427-447.

Reeves, C. W., Nicholls, A. R., \& McKenna, J. (2009). Stressors and Coping Strategies among Early and Middle Adolescent Premier League Academy Soccer Players: Differences According to Age. Journal of Applied Sport Psychology, 21(1), 3148.

Roe, C., \& Parker, A. (2016). Sport, Chaplaincy and Holistic Support: The Elite Player Performance Plan (EPPP) in English Professional Football. Practical Theology, 9(3), 169-182.

Rongen, F., McKenna, J., Cobley, S., \& Till, K. (2018). Are youth sport talent identification and development systems necessary and healthy? Sports Medicine - Open, 4, 18.

Ryan, R. M., \& Deci, E. L. (2000). Self-Determination Theory and the facilitation of intrinsic motivation, social development, and well-being. American Psychologist, 68-78. 
Sabato, T. M., Walch, T. J., \& Caine, D. J. (2016). The elite young athlete: strategies to ensure physical and emotional health. Open Access Journal of Sports Medicine, 7, 99-113.

Sagar, S. S., Busch, B. K., \& Jowett, S. (2010). Success and Failure, Fear of Failure, and Coping Responses of Adolescent Academy Football Players. Journal of Applied Sport Psychology, 22(2), 213-230.

Schnell, A., Mayer, J., Diehl, K., Zipfel, S., \& Thiel, A. (2014). Giving everything for athletic success! - Sports-specific risk acceptance of elite adolescent athletes. Psychology of Sport \& Exercise, 15, 165-172.

Sheldon, K., \& Hilpert, J. (2012). The balanced measure of psychological needs (BMPN) scale: An alternative domain general measure of need satisfaction. Motivation \& Emotion, 36(4), 439-451.

Stambulova, N. (2003). Symptoms of a crisis-transition: A grounded theory study. In N. Hassmén (Ed.), SIPF Yearbook 2003 (pp. 97-109). Örebro: Örebro University Press.

Tabachnick, B. \& Fidell, L.S. (2007) Using Multivariate Statistics (5th ed.).Boston: Pearson Education, Inc.

Tamminen, C. \& Braun, K.A. (2017). Psychological considerations of adolescents in sport and performance. In Oxford Research Encyclopedia of Psychology. Oxford University Press: DOI: 10.1093/acrefore/9780190236557.013.178

Tasiernski, T., Kennedy, P., Gardner, B., \& Blaikley, R. (2004) Athletic identity and sports participation in people with spinal cord injury. Adapted Physical Activity Quarterly, 21, 364-378.

Taylor, I. M., \& Bruner, M. W. (2012). The social environment and developmental experiences in elite youth soccer. Psychology of Sport \& Exercise, 13, 390-396.

Tears, C., Chesterton, P., \& Wijnbergen, M. (2018). The elite player performance plan: the impact of a new national youth development strategy on injury characteristics in a premier league football academy. Journal of Sports Sciences, 36(19), 2181-2188.

The KIDSCREEN Group Europe (2006). The KIDSCREEN Questionnaires Quality of life questionnaires for children and adolescents Handbook. Lengerich, Germany: Pabst Science Publishers.

The Premier League. (2011). Elite Player Performance Plan (EPPP). 
Verkooijen, K. T., van Hove, P., \& Dik, G. (2012). Athletic identity and well-being among young talented athletes who live at a Dutch elite sport center. Journal of Applied Sport Psychology, 24(1), 106-113.

Visek, A., Hurst, J., Maxwell, J., \& Watson, J. (2008) A cross-cultural psychometric evaluation of the athletic identity measurement scale. Journal of Applied Sport Psychology, 20, 473-480.

Weiss, C. (1998). Evaluation: Methods for studying programs and policies. (2 ${ }^{\text {nd }}$ ed). Upper Saddle River, NJ: Prentice Hall.

Wiersma, L. D. (2000). Risks and benefits of youth sport specialization: Perspectives and recommendations. Pediatric Exercise Science, 12(1), 13-22.

Williams, A. M., \& Reilly, T. (2000). Talent identification and development in soccer. Journal of Sports Sciences, 18(9), 657-667. 
Table 1: Questionnaires and subscales mapped onto commonly raised psychosocial concerns.

Concern

REST-Q

BMPN

KIDSCREEN-27

AIMS

- High perceived pressure/stress and low recovery (e.g., Bergeron et al. 2015; DiFiori et al., 2014, Sabato et al., 2016)

- $\quad$ Lack of energy and physical wellbeing (e.g., Bergeron et al., 2015; DiFiori et al., 2014)

- Lowered mood and psychological well-being (e.g., Bergeron et al., 2015 Sabato; Miller \& Kerr, 2002)

- Reduced sense of competence (e.g., Bergeron et al., 2015; Sabato et al., 2016; Wiersma, 2000)

- $\quad$ Reduced sense of belonging and connectedness (e.g., Bergeron et al., 2015; Miller \& Kerr, 2002)

- Reduced sense of autonomy and 'free' time (e.g. Bergeron et al., 2015; Sabato et al., 2016)

- Reduced educational enjoyment and engagement (e.g., Miller \& Kerr, 2002; Cosh \& Tully, 2014)

- Strong athletic identity (e.g.,

Miller \& Kerr, 2002; Sabato et al.

\section{General Stress, General}

Stress, Sport Specific recovery

General Recovery, Sport Specific Recovery

2016)
HRQoL - Physical well-being

HRQoL-Psychological wellbeing

Competence satisfaction competence thwarting

Relatedness satisfaction, relatedness thwarting

Autonomy satisfaction, autonomy thwarting
HRQoL - Social support and peers

HRQoL - Autonomy and parent relationships

HRQOL - School environment

Table Notes: REST-Q sport = Recovery and Stress Questionnaire for Sport (Kellmann, Altenburg, Lormes, \& Steinacker, 2001), BMPN = Balanced Measure of Psychological Needs (Sheldon \& Hilpert, 2012), KIDSCREEN-27 = Health related quality of life questionnaire (the KIDSCREEN group, 2006), AIMS = Athletic Identity Measurement Scale (Brewer \& Cornelius, 2001) 
Table 2. Psychosocial outcomes reported by academy soccer players and school pupils at four different time points.

\begin{tabular}{|c|c|c|c|c|c|c|c|c|c|}
\hline \multirow[t]{2}{*}{ Variable } & \multirow{2}{*}{$\begin{array}{l}\text { Scale } \\
\text { range }\end{array}$} & \multicolumn{2}{|c|}{$T 1$} & \multicolumn{2}{|c|}{$T 2$} & \multicolumn{2}{|c|}{$T 3$} & \multicolumn{2}{|c|}{$T 4$} \\
\hline & & Academy & School & Academy & School & Academy & School & Academy & School \\
\hline \multicolumn{10}{|l|}{ Stress \& Recovery } \\
\hline General stress $\#$ & $0-6$ & $1.46 \pm 0.62$ & $1.60 \pm 0.47$ & $1.29 \pm 0.64$ & $1.69 \pm 0.57$ & $1.27 \pm 0.61$ & $1.39 \pm 0.54$ & $1.35 \pm 0.66$ & $1.37 \pm 0.49$ \\
\hline General recovery ${ }^{ \pm}$ & $0-6$ & $3.62 \pm 0.82$ & $3.73 \pm 0.73$ & $3.75 \pm 0.82$ & $3.76 \pm 0.65$ & $3.86 \pm 0.76$ & $3.95 \pm 0.71$ & $3.80 \pm 0.72$ & $4.03 \pm 0.65$ \\
\hline Sport Stress ${ }^{\#, \pm}$ & $0-6$ & $1.78 \pm 0.78$ & $1.63 \pm 0.69$ & $1.56 \pm 0.81$ & $1.64 \pm 0.67$ & $1.41 \pm 0.77$ & $1.39 \pm 0.84$ & $1.41 \pm 0.77$ & $1.44 \pm 0.68$ \\
\hline Sport Recovery ${ }^{ \pm}$ & $0-6$ & $3.55 \pm 0.81$ & $3.53 \pm 0.83$ & $3.80 \pm 0.99$ & $3.69 \pm 0.85$ & $3.70 \pm 0.89$ & $3.82 \pm 0.85$ & $3.84 \pm 0.86$ & $3.95 \pm 0.84$ \\
\hline \multicolumn{10}{|l|}{ Need satisfaction } \\
\hline Relatedness satisfaction & $1-5$ & $3.94 \pm 0.72$ & $3.80 \pm 0.63$ & $3.97 \pm 0.64$ & $3.74 \pm 0.66$ & $4.07 \pm 0.54$ & $3.76 \pm 0.58$ & $3.89 \pm 0.76$ & $3.85 \pm 0.64$ \\
\hline Relatedness dissatisfaction & $1-5$ & $1.79 \pm 0.64$ & $1.88 \pm 0.63$ & $1.91 \pm 0.76$ & $1.92 \pm 0.60$ & $1.96 \pm 0.82$ & $2.01 \pm 0.68$ & $1.89 \pm 0.66$ & $1.75 \pm 0.69$ \\
\hline Autonomy satisfaction & $1-5$ & $3.92 \pm 0.55$ & $3.86 \pm 0.57$ & $3.92 \pm 0.55$ & $3.79 \pm 0.52$ & $3.91 \pm 0.55$ & $3.81 \pm 0.58$ & $3.75 \pm 0.59$ & $3.79 \pm 0.54$ \\
\hline Autonomy dissatisfaction & $1-5$ & $2.38 \pm 0.71$ & $2.57 \pm 0.74$ & $2.16 \pm 0.88$ & $2.31 \pm 0.71$ & $2.28 \pm 0.86$ & $2.27 \pm 0.74$ & $2.13 \pm 0.80$ & $2.27 \pm 0.72$ \\
\hline Competence satisfaction & $1-5$ & $3.55 \pm 0.54$ & $3.76 \pm 0.69$ & $3.84 \pm 0.50$ & $3.78 \pm 0.65$ & $3.74 \pm 0.54$ & $3.83 \pm 0.58$ & $3.56 \pm 0.53$ & $3.84 \pm 0.52$ \\
\hline Competence dissatisfaction & $1-5$ & $2.18 \pm 0.66$ & $2.30+0.68$ & $2.02 \pm 0.75$ & $2.21 \pm 0.77$ & $2.21 \pm 0.86$ & $2.18 \pm 0.74$ & $2.20 \pm 0.62$ & $2.09 \pm 0.84$ \\
\hline \multicolumn{10}{|l|}{ Health related quality of life } \\
\hline Physical well-being & $1-5$ & $48.24 \pm 7.04$ & $50.65 \pm 9.24$ & $51.50 \pm 7.54$ & $49.71 \pm 8.73$ & $50.35 \pm 8.07$ & $52.80 \pm 9.43$ & $51.99 \pm 9.11$ & $51.94 \pm 8.56$ \\
\hline Psychological well-being & $1-5$ & $50.05 \pm 7.46$ & $48.94 \pm 5.31$ & $52.71 \pm 9.80$ & $49.27 \pm 5.24$ & $51.10 \pm 9.50$ & $49.27 \pm 7.75$ & $50.70 \pm 7.23$ & $51.67 \pm 7.37$ \\
\hline Autonomy + parent relation & $1-5$ & $46.55 \pm 7.55$ & $45.98 \pm 7.15$ & $50.41 \pm 9.66$ & $47.10 \pm 7.31$ & $48.51 \pm 7.36$ & $47.75 \pm 8.57$ & $48.96 \pm 8.82$ & $48.06 \pm 8.71$ \\
\hline Peers \& Social support & $1-5$ & $45.20 \pm 6.33$ & $48.64 \pm 8.09$ & $47.18 \pm 12.14$ & $49.00 \pm 9.04$ & $45.33 \pm 9.20$ & $48.57 \pm 8.04$ & $45.04 \pm 7.80$ & $48.06 \pm 8.30$ \\
\hline School Environment ${ }^{\S}$ & $1-5$ & $46.74 \pm 7.47$ & $45.24 \pm 6.80$ & $46.72 \pm 9.43$ & $47.06 \pm 6.96$ & $46.32 \pm 9.33$ & $45.55 \pm 7.75$ & $45.00 \pm 8.11^{\S}$ & $50.28 \pm 8.54$ \\
\hline \multicolumn{10}{|l|}{ Athletic Identity } \\
\hline Social identity & $1-7$ & $5.69 \pm 0.69$ & $5.46 \pm 0.91$ & $5.96 \pm 0.88$ & $5.53 \pm 1.20$ & $5.92 \pm 0.70$ & $5.61 \pm 1.09$ & $5.93 \pm 0.81$ & $5.46 \pm 1.25$ \\
\hline Exclusivity* & $1-7$ & $5.85 \pm 0.96$ & $4.78 \pm 1.38$ & $6.01 \pm 0.87$ & $4.98 \pm 1.42$ & $6.13 \pm 0.83$ & $5.03 \pm 1.18$ & $6.07 \pm 0.92$ & $5.02 \pm 1.21$ \\
\hline Negative affectivity & $1-7$ & $5.74 \pm 1.23$ & $5.26 \pm 1.15$ & $6.07 \pm 0.88$ & $5.39 \pm 1.38$ & $5.68 \pm 1.49$ & $5.48 \pm 1.38$ & $5.94 \pm 0.95$ & $5.35 \pm 1.43$ \\
\hline Total athletic identity* & $1-7$ & $40.24 \pm 4.57$ & $36.6 \pm 5.72$ & $42.06 \pm 4.73$ & $37.74 \pm 6.00$ & $41.38 \pm 4.46$ & $38.05 \pm 6.22$ & $41.82 \pm 4.54$ & $37.98 \pm 6.09$ \\
\hline
\end{tabular}

Table notes: Soccer players $n=33$, school pupils $n=44$. Data is presented as mean \pm SD. $*$ indicates significant main effect of group soccer players different

to school pupils at all time points, ${ }^{\#} \&{ }^{ \pm}$indicate significant main effects of time T1 different to T3 \& T4 respectively, ${ }^{\S}$ indicates a significant group*time interaction effect at T4. Significance accepted at $\mathrm{p}<0.0125$ for all analyses. 
Supplementary Table: Cronbach's alpha scores for each subscale of the psychosocial questionnaires used.

\begin{tabular}{lccccc}
\hline \multicolumn{1}{c}{ Variable } & Scale range & $\boldsymbol{T 1}$ & $\boldsymbol{T 2}$ & $\boldsymbol{T 3}$ & $\boldsymbol{T 4}$ \\
& & $\boldsymbol{a}$ & $\boldsymbol{a}$ & $\boldsymbol{a}$ & $\boldsymbol{a}$ \\
\hline Stress \& Recovery & & & & & \\
General stress & $0-6$ & .74 & .83 & .82 & .81 \\
General recovery & $0-6$ & .71 & .76 & .77 & .74 \\
Sport Stress & $0-6$ & .79 & .73 & .85 & .82 \\
Sport Recovery & $0-6$ & .88 & .91 & .82 & .91 \\
Need satisfaction and Thwarting & & & & & \\
Relatedness satisfaction & $1-5$ & .68 & .73 & .66 & .76 \\
Relatedness dissatisfaction & $1-5$ & .56 & .61 & .52 & .64 \\
Autonomy satisfaction & $1-5$ & .74 & .68 & .54 & .74 \\
Autonomy dissatisfaction & $1-5$ & .41 & .65 & .68 & .59 \\
Competence satisfaction & $1-5$ & .58 & .54 & .48 & .66 \\
Competence dissatisfaction & $1-5$ & .51 & .60 & .47 & .46 \\
Need satisfaction & & & & & \\
Physical well-being & $1-5$ & .77 & .79 & .78 & .78 \\
Psychological well-being & $1-5$ & .78 & .82 & .73 & .72 \\
Autonomy + parent relation & $1-5$ & .79 & .76 & .80 & .83 \\
Peers \& Social support & $1-5$ & .72 & .83 & .80 & .76 \\
School environment & $1-5$ & .82 & .81 & .76 & .83 \\
Athletic Identity & & & & & \\
Social identity & $1-7$ & .56 & .81 & .77 & .83 \\
Exclusivity* & $1-7$ & .70 & .85 & .87 & .79 \\
Negative affectivity & $1-7$ & .42 & .54 & .69 & .62 \\
Total athletic identity* & $1-7$ & .70 & .80 & .79 & .83 \\
\hline
\end{tabular}




\section{Figure captions}

Figure 1: Combined perceived stress and recovery scores for academy players and agematched school pupils across four time points in an academic year.

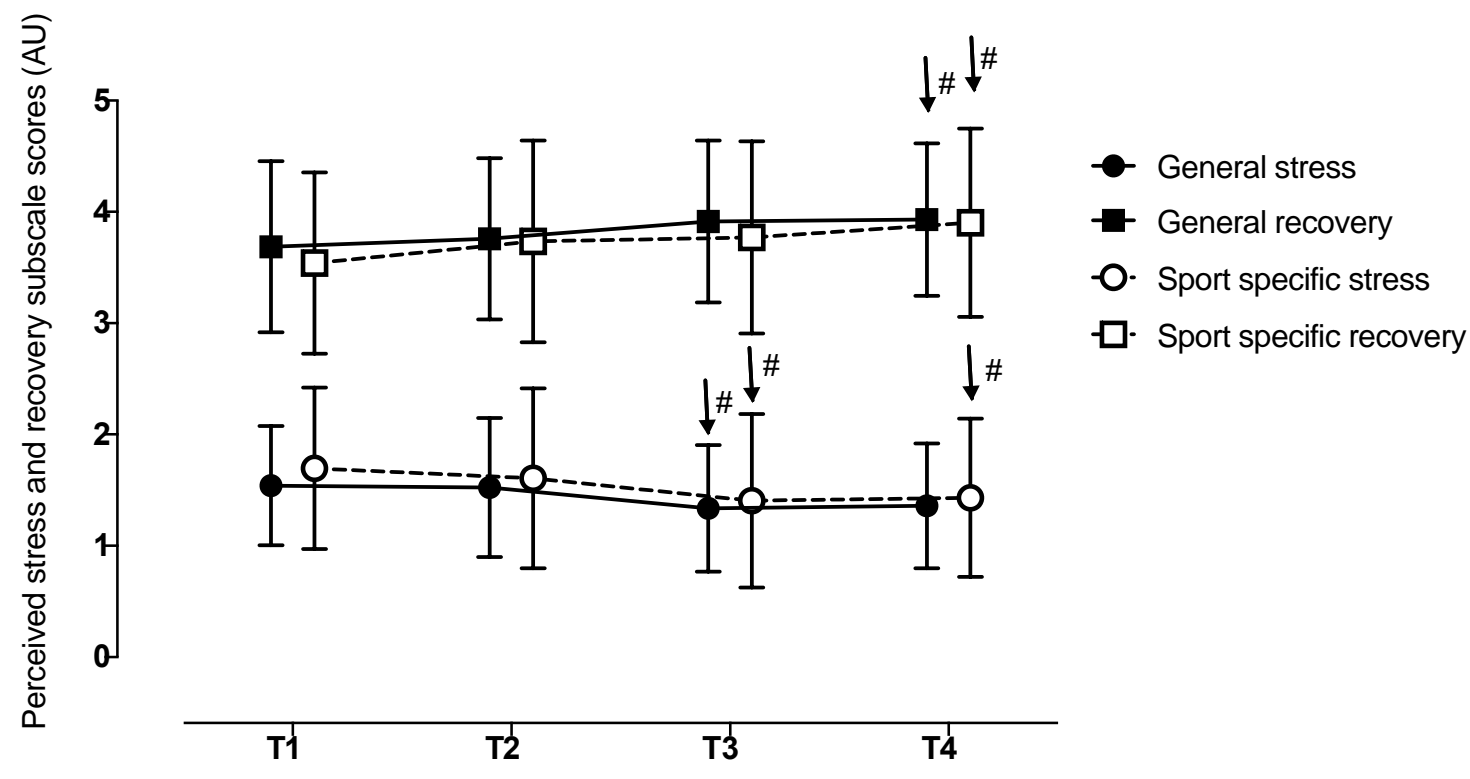

Figure note: \# indicates a significant time effect $(\mathrm{p}<0.0125)$. 
Figure 2: Comparison of academy players and age-matched school pupil perceptions of School Related Quality of Life subscale of the Health-Related Quality of Life Questionnaire

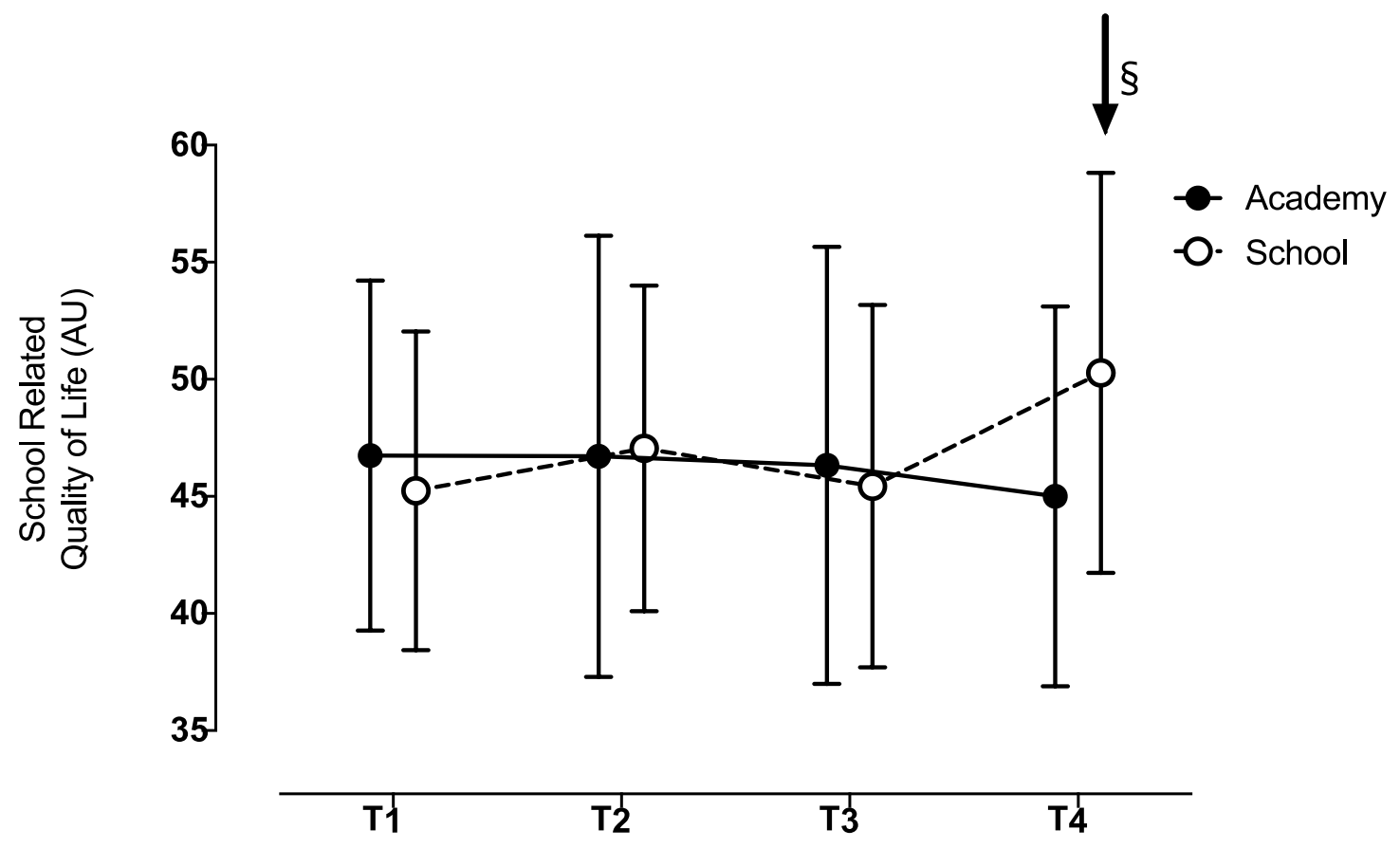

Figure Note: $\S$ indicates a significant group*time effect $(\mathrm{p}<0.0125)$ at the time point indicated. 
Figure 3: Comparison of athletic identity subscales between academy players and age matched school pupils.

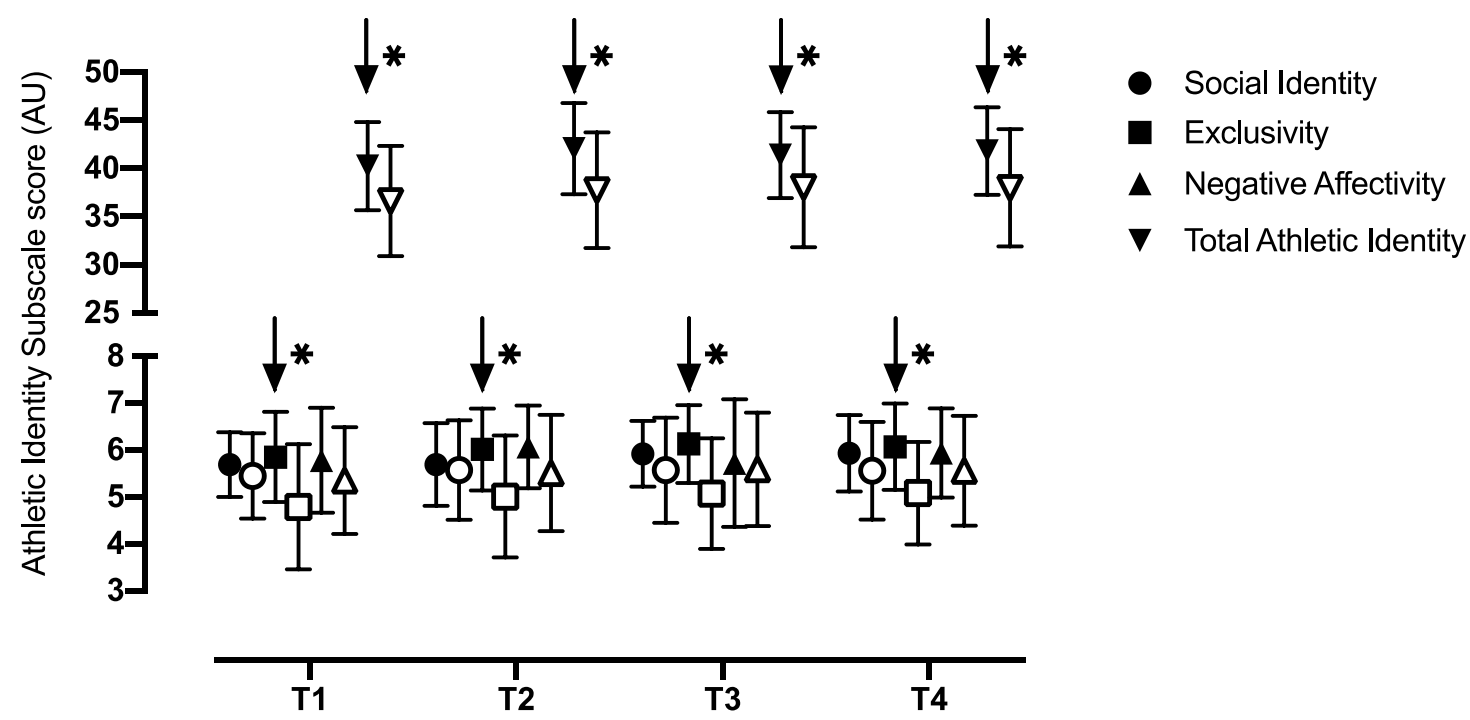

Figure Notes: Academy players are represented by filled shapes, and age-matched school pupils by hollow shapes. $*$ indicates a significant group effect $\mathrm{p}<0.0125$. 